\title{
SIZE LIMITS FOR EXPANDING LIGHT SOURCES James Terre11
}

Los Alamos Scientific Laboratory, Los Alamos, N. M. 87545, U. S. A.

Les fluctuations rapides des brillances optiques et radio observées dans les quasars, ainsi que dans les objets BL Lac, imposent des limites superrieures sévères sur les dimensions possibles de ces sources; contrairement a l'opinion répandue, ces limites ne sont pas notablement modifiées par l'hypothèse d'une source lumineuse en expansion rapide. Le conflit entre les limites de variation des dimensions des quasars et la surface minimale pouvant émettre une énergie suffisante, en supposant des distances cosmologiques, n'est pas résolu par une expansion relativiste.

Rapid fluctuation in radio and optical brightness is one of the most outstanding characteristics of quasars, as well as of BL Lac objects. In many papers on these fluctuations the authors have found it necessary to assume a relativistically-expanding light source in order to resolve the conflict between fluctuation size limits and the surface area needed for the radiation of enormous power, as required by the assumption of cosmological distance. It is the purpose of this paper to show that the widespread belief in this advantageous feature of relativistic expansion is misplaced, and that the size limits for fluctuating light sources are not essentially changed by relativistic expansion.

For a spherical source of radius $R$ which is not expanding, it can be shown that the rate of change of luminosity $L$, for arbitrary fluctuation in surface brightness, is subject to the 1 imit $\left|\frac{d L}{d t}\right| \leqq \frac{2 c L_{\max }}{R(1+z)}$, in which $\mathrm{L}_{\max }$ is the maximum luminosity which could be observed in the absence of retardation effects, and $z$ is the redshift (Terrel1 1966, 1967, $1976 a, b)$. 
As Rees (1966, 1967) has pointed out, relativistic expansion at speed $v$ can cause the source size to expand at apparent speed $\gamma v$, which can be much greater than the speed of 1 ight $c$, with $\gamma \equiv\left(1-v^{2} / c^{2}\right)^{-1 / 2}$. This effect can lead to a very rapidly-rising light pulse which will, however, still be subject to the same limit given above. More importantly, and contrary to widespread belief, relativistic expansion will not lead to very short light pulses (Terrel1 1968, 1976a,b). The pulse length is determined not by the rapid rise of light but by the possibly very large distance $R$ from the explosion center at which the expanding surface becomes optically thin.

As indicated in Figure 1, the surface is seen to become transparent at the center at time $T_{1}$; at time $T_{2}$ its apparent expansion speed begins to

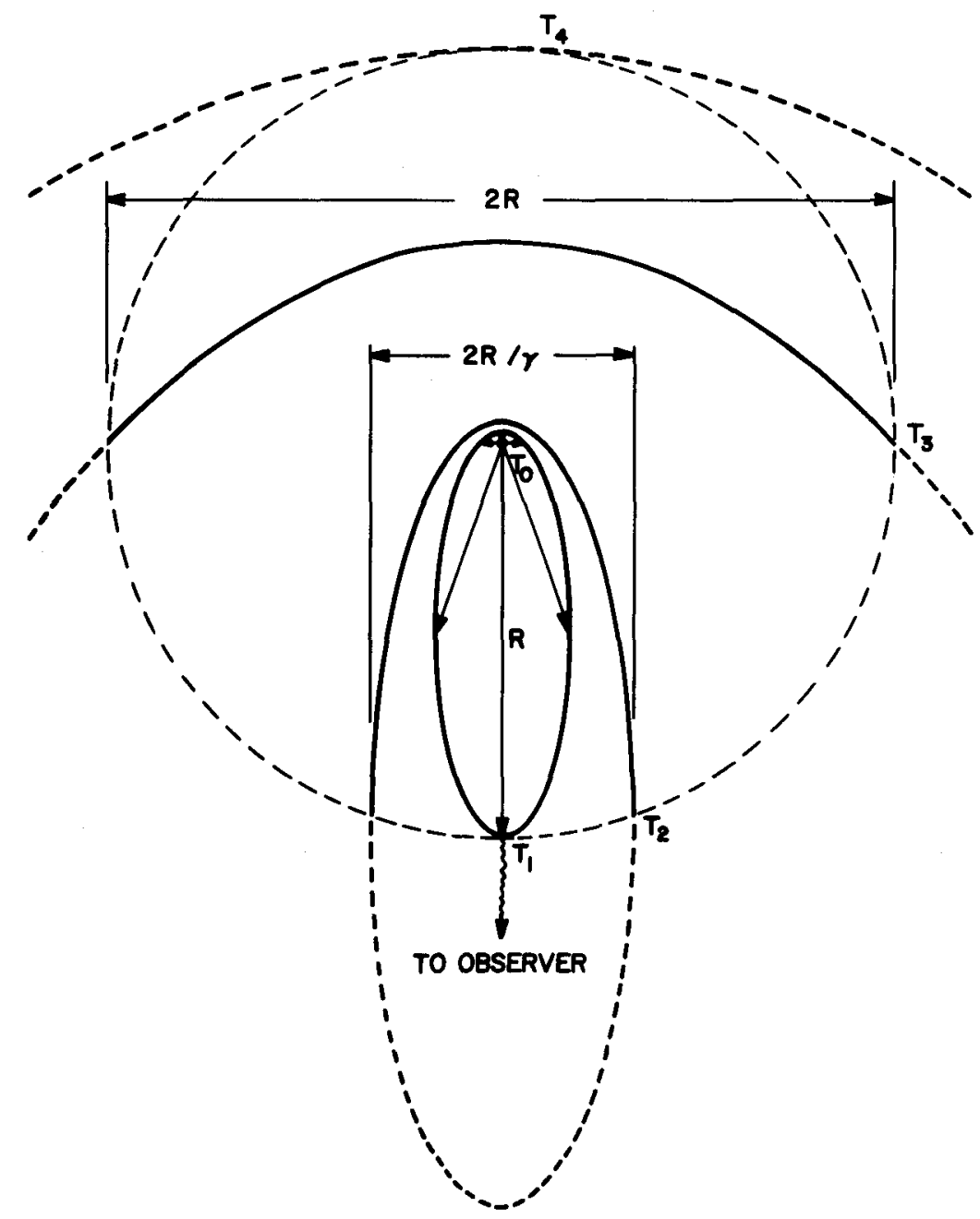

Fig. 1. Apparent shape, at various times, of a relativistically-expanding surface which becomes optically thin upon reaching radius $R$, for the case $\gamma=3$. 
slow; at time $T_{3}$ it reaches its maximum diameter $2 R$ and begins to shrink, until at time $\mathrm{T}_{4}$ it is no longer visible. It is assumed here for simplicity that the transition from optically thick to transparently thin occurs sharply at the distance R.

Figure 2 shows the light output from such an expanding source. If the total brightness is proportional to observed area, as shown by the heavy line, the light output has an effective pulse length of $1.67 \mathrm{R} / \mathrm{c}$. If the surface brightness, alternatively, is assumed to be that of a self-absorbed synchrotron radiation source, proportional to the square root of the Doppler shift factor for each point, the light pulse peaks earlier but has the even longer effective pulse length of $1.72 \mathrm{R} / \mathrm{C}$ in this example $(\gamma=5)$. The effective pulse length as defined by Terrell and 01 sen (1970, 1972) is the most significant measure of pulse length, and may be determined from power spectra even if individual outbursts cannot be separated (Terre11 1972).

Thus the effective pulse length for an expanding source is determined not by the initial rapid rise of intensity when the source is small but by the possibly very large value $R$ of the maximum source size. A good example

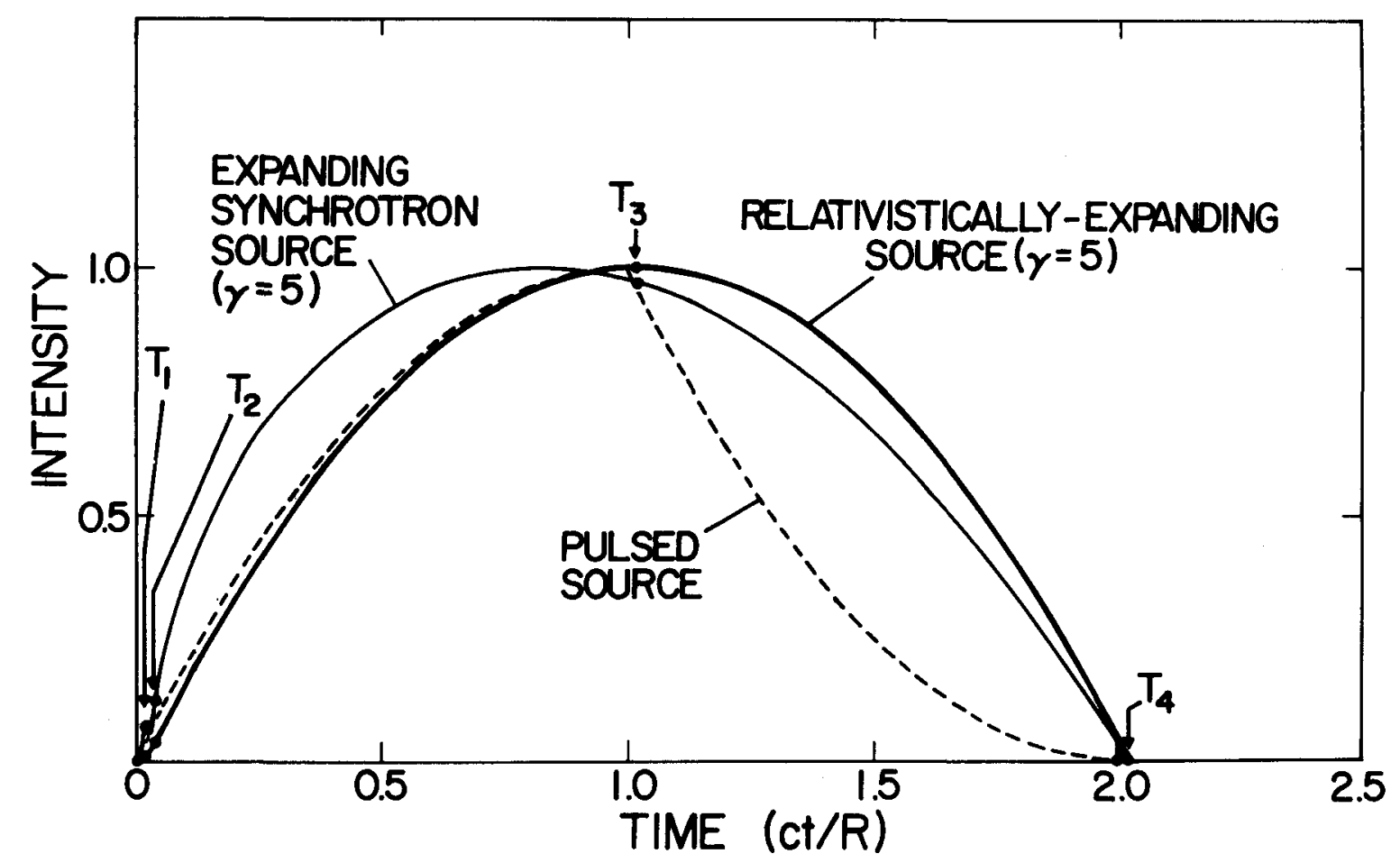

Fig. 2. Theoretical pulse shapes for a relativistically-expanding surface of maximum radius $R$, compared with a light pulse from a stationary source, also of radius $R$. 
is furnished by the explanation of supernova light curves by Morrison and Sartori (1966, 1969), in which an ultraviolet radiation front stimulates visible emission as it moves out at the speed of light $(\gamma=\infty)$, the effective pulse length of several months being determined by the maximum size of the emission region.

If a large non-expanding source of radius $R$ were simply turned on for a time $R / C$ and then off, the resulting light pulse, also shown in Figure 2 , would have an effective pulse length of $1.36 \mathrm{R} / \mathrm{c}$, smaller than the values just discussed, and the assumption of a shorter time on would lead to an even smaller value, the limit being $0.75 \mathrm{R} / \mathrm{C}$. Thus the effective pulse length $T_{e}$ gives an upper limit to the source size, $R \approx c T_{e}$, independently of whether the source is expanding relativistically or not.

No real advantage is then gained here by the assumption of relativistic expansion. The rapid fluctuations of brightness observed for many quasars and BL Lac objects still indicate, in the absence of known mechanisms for incredible surface brightness, that these objects cannot reasonably be at cosmological distance (Terrel1 1967, 1975).

The results on relativistic expansion briefly summarized here are given in more detail elsewhere (Terre11 1976b). This work has been supported by the U.S.E.R.D.A.

\section{REFERENCES}

Morrison, P., and Sartori, L. 1966, Phys. Rev. Letters, 16, 414-419.

------. 1969, Ap. J., 158, 541-570.

Rees, M. J. 1966, Nature, 211, 468-470.

-.--. 1967, M.N.R.A.S., 135, 345-360.

Terre11, J. 1966, Science, 154, 1281-1288.

-...-.. 1967, Ap. J., 147, 827-832.

-.---. 1968, Phys. Rev. Letters, 21, 637-641.

-----. 1972, Ap. J., 174, L35-L41.

-----. 1975, in Theories and Experiments in High Energy Physics (Proc.

Orbis Scientiae II, Coral Gables), ed. B. Kursunoglu, A. Perlmutter, and

S. M. Widmayer (New York: Plenum), pp. 457-473.

--.-. 1976a, Bu11. A.P.S., 21, 522.

--1976b, paper submitted to Astrophysical Journal (Letters).

Terrel1, J., and 01sen, K. H. 1970, Ap. J., 161, 399-413.

-...-.. 1972, in Proc. IAU Symposium №. 44, External Galaxies and Quasi

Stellar 0bjects, ed. D. S. Evans (Dordrecht: Reidel), pp. 179-187. 